\title{
Der Zusammenhang von Schuleinstellung und Qualität kind- und klassenbezogener pädagogischer Beziehungen von Lehrkräften
}

\author{
Sina Schürer $(\mathbb{D}) \cdot$ Stefanie van Ophuysen $(\mathbb{D})$ Sophie Michalke $\mathbb{D}$
}

Eingegangen: 1. Oktober 2020 / Überarbeitet: 6. April 2021 / Angenommen: 8. April 2021 / Online publiziert: 4. Mai 2021

(C) Der/die Autor(en) 2021

Zusammenfassung Eine positive affektive Einstellung gegenüber Schule als Ort des Lernens, Leistens und Zusammenlebens kann als zentrale Komponente des schulischen Wohlbefindens gelten. Die Sicherung schulischen Wohlbefindens ist neben der Förderung von Lernprozessen und -leistungen ein grundlegender Auftrag von Schule. Neben Merkmalen des individuellen Kindes und der Unterrichtsgestaltung hat sich insbesondere die Lehrkraft-Schüler/in-Beziehung als Prädiktor des Wohlbefindens herausgestellt. Die Lehrkraft-Schüler/in-Beziehung ist eine pädagogische Beziehung, die professionell-orientiert ist und zur Förderung eines positiven Schulerlebens ausgerichtet ist, indem 1) positive Interaktionen und Wertschätzung sowie 2) fachlich und emotionale Hilfestellung gewährleistet und 3) Mitbestimmung ermöglicht wird. Diese pädagogische Beziehung wird in einer sozialen Gruppe - der Klasse - ausgeübt, weshalb es sinnvoll ist, diesen Gruppenbezug einzubinden. So ergibt sich neben der Lehrkraft-Schüler/in-Beziehung auch eine Lehrkraft-KlassenBeziehung. In bisheriger Forschung wird der Gruppenbezug vernachlässigt oder durch Aggregierung von Individualbeziehungen nicht adäquat abgebildet. Die vorliegende Studie differenziert zwischen kindbezogener und klassenbezogener pädagogischer Beziehung. Es wird erwartet, dass sich eine höhere positive affektive Schuleinstellung zeigt, wenn die Beziehung der Lehrkraft zum individuellen Kind sowie zur Klasse als Ganzes vom Kind als positiv wahrgenommen wird. Im Rahmen einer standardisierten schriftlichen Befragung wurden Daten von 477 Schüler/ innen (Jahrgang 5/6) aus drei Gymnasien in Nordrhein-Westfalen erhoben. Unter Berücksichtigung der Mehrebenenstruktur können Effekte beider Beziehungsfacetten auf die Schuleinstellung regressionsanalytisch nachgewiesen werden. Weitere For-

Dr. S. Schürer $(\varangle) \cdot$ Prof. Dr. S. van Ophuysen $\cdot$ S. Michalke

Institut für Erziehungswissenschaft, WWU Münster, Georgskommende 33, 48143 Münster,

Deutschland

E-Mail: schuerer@uni-muenster.de 
schungsdesiderate werden aufgezeigt und Implikationen für die schulische Praxis werden diskutiert.

Schlüsselwörter Wohlbefinden · Schuleinstellung · Pädagogische Beziehungen · Lehrkraft-Schüler/in-Beziehung

\title{
Affective school attitudes and the quality of teachers' child- and class- related pedagogical relations
}

\begin{abstract}
A positive affective attitude towards school as a place of learning, achievement, and living together can be considered a central component of school wellbeing. In addition to the promotion of learning processes and performance, it is a fundamental mission of schools to ensure well-being at school. Along with individual child's characteristics and the way teachers organize lessons, the teacher-student relationship, in particular, has emerged as a predictor of well-being. The teacherstudent relationship is a pedagogical relationship that is professionally oriented and therefore, it is designed to promote a positive school experience by 1) ensuring positive interaction and appreciation, 2) providing professional and emotional support, and 3) enabling co-determination. This pedagogical relationship is practiced in a social group - the class - which makes necessary to include this group reference. Thus, in addition to the teacher-pupil relationship, a teacher-class relationship also results. Previous research has neglected the group relationship or has not adequately operationalized it by aggregating individual relationships. Hence, this study differentiates between child-related and class-related pedagogical relationships. We expect that a positive affective attitude to be higher if the teacher's relationship to the individual child and the class as a whole is perceived positively by the child in terms of the mentioned characteristics. In a standardized survey, data were collected from 477 students (year 5/6) from three high schools in North Rhine-Westphalia (Germany). Considering the multilevel-structure effects of both relationship, facets on school attitudes are demonstrated using regression analysis. We discuss further research desiderata and the implications for school practice.
\end{abstract}

Keywords Well-being - Attitude toward school - Pedagogical relationship · Teacher-student-relation

\section{Einleitung}

Dass Kinder gerne zur Schule gehen und eine positive Einstellung gegenüber dem Lernen und der Schule entwickeln, ist neben dem Anspruch an einen möglichst hohen Lernerfolg eine unbestrittene Forderung an die Institution Schule (Hagenauer 2011). In vielen Schulen ist bereits im Leitbild formuliert, dass die Schule Wert darauflegt, eine Umgebung zu schaffen, in der sich Schüler/innen wohlfühlen (Hascher und Lobsang 2004). Auch bei der Auszeichnung von Schulen mit dem Deutschen Schulpreis wird das Wohlbefinden der Schüler/innen als wichtiges Kriterium berücksichtigt (z.B. Veith 2016). Insgesamt wird schulisches Wohlbefinden als eine 
Grundbedingung gelingender Lernprozesse gesehen (Fend und Sandmeier 2004) und dient gerade auch in inklusiven schulischen Kontexten als wichtiger Indikator schulischer Qualität (z. B. Külker et al. 2017).

Für die schulische Praxis ist daher die Frage relevant, welche Merkmale Einfluss auf das schulische Wohlbefinden nehmen. Dabei können Prädiktoren auf Ebene des Kindes (z. B. Leistungsniveau, Leistungszufriedenheit, Selbstwirksamkeit, Kontakte zu Peers) und auf Ebene der Lehrkraft (z. B. Unterrichtsgestaltung, Bezugsnormorientierung, Lehrkraft-Schüler/in-Beziehung) in den Blick genommen werden (Hascher 2004). In diesem Beitrag legen wir den Fokus auf den Zusammenhang zwischen einer positiven Schuleinstellung als zentrale Komponente des schulischen Wohlbefindens und der Qualität der pädagogischen Beziehung. Lehrkräfte beziehen sich in ihrer Interaktion sowohl auf das individuelle Kind als auch auf die Klasse als Ganzes. Daher differenzieren wir in unserem Beitrag zwischen der Qualität der Beziehung, die sich auf das Kind und der Beziehungsqualität zur Schulklasse bezieht und gehen der Frage nach, ob beide Facetten der Beziehungsqualität einen eigenständigen Beitrag zur Schuleinstellung des Kindes liefern.

\section{Theoretischer Hintergrund und Forschungsstand}

\subsection{Das Konzept des schulischen Wohlbefindens}

Wohlbefinden ist ein Konstrukt, das seit den 1960er-Jahren (nicht nur) in der psychologischen Forschung von Bedeutung ist, für das aber immer noch keine allgemein anerkannte Theorie oder eine einheitliche Definition besteht (Hascher et al. 2018). Hascher (2004) sieht drei Aspekte als kleinsten gemeinsamen Nenner der vielfältigen Zugänge: Wohlbefinden umfasst sowohl affektive als auch kognitive Aspekte, es müssen positive wie negative Emotionen und Bewertungen berücksichtigt werden und das Empfinden von Freude ist zentral.

Weiterhin steht außer Frage, dass Wohlbefinden bereichsspezifisch betrachtet werden sollte (Becker 1994). So unterscheiden beispielsweise Diener et al. (1999) zwischen der Zufriedenheit im Beruf, in der Familie oder in der Freizeit. Für Kinder und Jugendliche ist der Bereich der Schule ein zentraler Lebensbereich, der allein schon quantitativ einen erheblichen Anteil ihrer Lebenszeit ausmacht. Entsprechend nimmt das schulische Wohlbefinden von Schüler/innen in den Bildungswissenschaften und der Pädagogischen Psychologie inzwischen einen wichtigen Stellenwert ein, doch auch hier wird der Begriff sehr unterschiedlich konzeptualisiert (Serke 2019). Für unsere Arbeit rekurrieren wir auf eine Definition von Hascher (2004), die Wohlbefinden konkretisiert als einen „Gefühlszustand [...], bei dem positive Emotionen und Kognitionen gegenüber der Schule, den Personen in der Schule und dem schulischen Kontext bestehen und gegenüber negativen Emotionen und Kognitionen dominieren“ (S. 150).

Für die empirische Untersuchung des schulischen Wohlbefindens wird häufig auf das mehrdimensionale Modell von Hascher (2004) zurückgegriffen. Eine der sechs Komponenten des Modells stellt die Freude in der Schule dar, beispielsweise durch das Erleben sozialer Einbindung, Anerkennung oder Mitbestimmungsmöglichkei- 
ten. Weiterhin gelten die Abwesenheit von Sorgen sowie das Fehlen körperlicher Beschwerden und sozialen Problemen als weitere Komponenten des schulischen Wohlbefindens. Die genannten Komponenten beziehen sich auf konkrete Erfahrungen des Schülers/der Schülerin. Die Abwesenheit negativer Gefühle wie Angst, Besorgnis oder Scham ermöglicht eine bessere Konzentration auf das Lernen. Zahlreiche Studien belegen entsprechend einen positiven Zusammenhang von positiven schulbezogenen Emotionen, bzw. dem Fehlen negativer Emotionen und Sorgen mit Schulleistungen und Schulerfolg (z. B. Beisenherz 2005; Czerwenka et al. 1990; Eder 1995; Fend 1997; Fend und Sandmeier 2004; Hascher 2004; Hascher und Edlinger 2009; Helmke 1993; Wagner und Valtin 2003). Hascher und Hagenauer (2011) sprechen daher auch von der Bildungsfunktion des schulischen Wohlbefindens.

Nach Hascher (2004) setzt schulisches Wohlbefinden jedoch nicht nur voraus, dass schulbezogene Freude das negative Erleben von Sorgen, körperlichen Beschwerden und sozialen Problemen dominiert, sondern gleichzeitig sollte die Schülerin/der Schüler sich selbst als eine Person wahrnehmen, die sich mit der Schule identifiziert und den schulischen Anforderungen gewachsen ist. Ebenso muss Schule für die Schülerin/den Schüler mit überdauernden positiven Kognitionen und Emotionen verbunden sein. Hascher bezeichnet diese beiden Modellkomponenten als schulischen Selbstwert und positive Schuleinstellung.

Eine positive Schuleinstellung basiert auf wiederholten positiven Erfahrungen in konkreten schulischen Situationen. Die Gestaltung von Schule derart, dass das Erleben von Freude ermöglicht und gleichzeitig Sorgen, körperliche Beschwerden und soziale Probleme geringgehalten werden, ist entsprechend sowohl ein Ziel für sich, als auch ein Weg, um eine positive Einstellung gegenüber der Schule und dem Lernen zu erreichen. Die Einstellung gegenüber der Schule stellt eine überdauernde Bewertungstendenz dar, die sich in Überzeugungen (z. B. wichtig/unwichtig; förderlich/ schädlich), Gefühlen (angenehm/unangenehm) und Verhaltensweisen (aufsuchen/ vermeiden) manifestiert. Sie stellt ein relativ stabiles Merkmal von Schülerinnen und Schülern dar.

Vermutlich nicht zuletzt aufgrund dieser relativen Unabhängigkeit von tagesaktuellen, situationsspezifischen Schwankungen ist die Schuleinstellung eine in empirischen Studien häufig genutzte Komponente des schulischen Wohlbefindens. Dabei dominieren meist die affektiven Anteile der Einstellung, was oft in der Nutzung von Begriffen wie Schulfreude oder Schulunlust resultiert. Auch wir werden in unserer Studie auf die (affektive) Schuleinstellung im Sinne einer relativ stabilen Komponente des schulischen Wohlbefindens fokussieren. Wenn explizit die positive Ausprägung der affektiven Einstellung gegenüber Schule als Ort des Lernens, Leistens und Zusammenlebens gemeint ist, sprechen auch wir von Schulfreude, wohlwissend, dass damit eine begriffliche Unschärfe hinsichtlich der Komponente der schulbezogenen Freude aus dem Modell von Hascher einhergeht. Wir erachten diese Unschärfe als hinnehmbar, da die Grenze zwischen dem konkreten Erleben (erfragt beispielsweise durch Items, die mit den Worten „Kam es in den letzten Wochen vor, dass ...“ eingeleitet werden) und der daraus letztlich resultierenden Einstellung fließend ist. So finden sich in empirischen Studien die höchsten Korrelationen zwischen diesen beiden Modellkomponenten (z. B. Hascher et al. 2011). 


\subsection{Prädiktoren von Wohlbefinden und Schuleinstellung}

Auch in der Literatur werden die Begriffe Wohlbefinden, positive Schuleinstellung und Schulfreude (zum Teil auch Schulzufriedenheit oder Schulunlust) häufig synonym genutzt bzw. nicht klar voneinander abgegrenzt. Im folgenden Überblick über empirische Studien wird daher auf eine klare Trennung zwischen den Konstrukten verzichtet.

Auf Ebene des individuellen Kindes zeigt sich, dass die Schuleinstellung abhängig vom Geschlecht ist. Mädchen weisen eine höhere Schulfreude auf als Jungen (z. B. Harazd und Schürer 2006; Hascher et al. 2011; Kirsch und Hansen 2002; Primas 2014; Schwab et al. 2015; Verkuyten und Thijs 2002; Walsen 2013). Ebenfalls hat das Alter der Schüler/innen einen Einfluss auf ihre Schuleinstellung. Sind in der Grundschule die Emotionen gegenüber der Schule noch überwiegend positiv, so sinkt die Schulfreude im Laufe der Schulzeit deutlich (van Ophuysen 2008; Czerwenka et al. 1990). Bereits im Laufe der Grundschulzeit kommt es zu einer Abnahme positiver Emotionen (z. B. Hascher et al. 2011; Mayr und Ulich 2002; Pupeter und Hurrelmann 2013). In Bezug auf den Migrationshintergrund ergeben sich widersprüchliche Befunde. In einigen Studien findet sich eine höhere Schulfreude bei Schüler/innen mit Migrationshintergrund (z. B. Rauer und Schuck 2003; Stanat und Christensen 2006; Verkuyten und Thijs 2002), in anderen Studien liegen keine statistisch bedeutsamen Unterschiede zwischen Schüler/innen mit und ohne Migrationshintergrund vor (z. B. Harazd und Schürer 2006; Kohl et al. 2011; Okun et al. 1990). Hinsichtlich der Schulleistung werden teils positive wenngleich schwache Zusammenhänge (z. B. Fend und Sandmeier 2004; Hascher 2004) berichtet. Andere Studien finden keinen Zusammenhang mit Leistungsvariablen (z. B. Lee 2016).

Auf Ebene der Lehrkraft zeigt sich, dass gut strukturierter und schüler/innzentrierter Unterricht sowie als gerecht wahrgenommenes Lehrkräftehandeln in positivem Zusammenhang mit der Schuleinstellung stehen (Eder 2007; Gläser-Zikuda und Fuß 2004; Hascher 2004). Hascher (2004) hebt den Sozialkontakt mit den Lehrpersonen als bedeutsame Quelle der Schuleinstellung hervor. Damit wird gleichzeitig die Relevanz des Klassenklimas für die Entwicklung einer positiven Schuleinstellung deutlich. Klassenklima wird definiert als die subjektive Wahrnehmung von Schulumweltmerkmalen durch die individuellen Schüler/innen (z. B. Eder 2002; von Saldern 1987) und die sozialen Beziehungen zwischen Lehrkräften und Schüler/innen stellen gerade ein solches Schulumweltmerkmal dar (Eder 2002). Insgesamt konnten wiederholt Zusammenhänge der Schulfreude mit dem Klassenklima nachgewiesen werden (z. B. Grewe 2017). Mit Blick auf die empirischen Befunde ist dabei besonders bemerkenswert, dass Zusammenhänge des Lehrkrafthandelns mit der Schuleinstellung des Kindes selbst bei sehr unterschiedlichen Operationalisierungen und methodischen Settings nachweisbar waren (Aldridge et al. 2016; Czerwenka et al. 1990; Fend und Sandmeier 2004; Hascher und Lobsang 2004; van Petegem et al. 2008). Sowohl in standardisierten schriftlichen Befragungen als auch in qualitativen Analysen von Schüler/innen-Aufsätzen oder Interviews mit Schüler/innen zeigte sich eine höhere Schulfreude bei Kindern, die eine fürsorgliche, sozial-integrative Lehrperson erleben, die ihnen Raum für selbstbestimmtes Handeln und Verantwortungsübernahme gewährt. 


\subsection{Pädagogische Beziehungen}

Eine Beziehung entsteht aus und manifestiert sich in wechselseitig aufeinander bezogenen Interaktionen von mindestens zwei Personen. Von einer pädagogischen Beziehung sprechen wir, wenn es sich um die Beziehung zwischen einer Person, die professionell im pädagogischen Kontext tätig ist, und einer oder mehrerer anderer Personen, auf die das pädagogische Handeln ausgerichtet ist, handelt. In diesem Sinne handelt es sich nach unserem Verständnis bei jeder (professionell orientierten) Beziehung zwischen Lehrkraft und Schüler/in um eine pädagogische Beziehung, wobei diese jedoch von sehr unterschiedlicher Qualität sein kann (Prengel 2019). Die Qualität der Beziehung zwischen Lehrkraft und Kind hängt von beiden Akteur/ innen ab. Dennoch muss aufgrund der Asymmetrie der Beziehung der lehrkraftseitigen Interaktionsgestaltung, also der Art und Weise, wie die Lehrkraft das individuelle Kind wahrnimmt und anspricht, besondere Bedeutung beigemessen werden.

In umfangreichen Forschungsarbeiten im Bereich der Lehrkraft-Schüler/in-Beziehung hat Robert C. Pianta mit seiner Arbeitsgruppe die Bedeutung emotional unterstützender Beziehungen zwischen Lehrkraft und Schüler/in für motivationale und akademische Kriterien herausgearbeitet. Unterschieden werden insbesondere drei Facetten, welche je nach Ausprägung für die unterschiedliche Qualität pädagogischer Beziehungen verantwortlich sein können: (1) eine positive Interaktion, die durch geteilte positive Affekte, Anerkennung, Wertschätzung und respektvolle Kommunikation aufgebaut wird, (2) Unterstützung, die sich äußert, indem fachliche und emotional-soziale Bedürfnisse der Kinder erkannt und die jeweils angemessene Hilfestellung rechtzeitig gewährt wird und (3) Mitbestimmung, indem Schüler/ innen ermutigt werden, ihre Ideen und Meinungen zu äußern und diese gehört sowie beachtet werden (Hamre et al. 2013; Ruzek et al. 2016). Diese Facetten der Beziehungsqualität wurden aus motivationspsychologischen Überlegungen abgeleitet und stehen in enger Relation zu den drei zentralen Bedingungen (soziale Eingebundenheit, Kompetenz- und Autonomieerleben), die nach der Selbstbestimmungstheorie von Deci und Ryan (2000) als Kernmerkmale eines motivierenden, lern- und leistungsförderlichen schulischen Umfelds gelten (Ruzek et al. 2016). Eine positive Interaktion beinhaltet neben einem freundlichen Ton und positiven Inhalten der Kommunikation auch das Bekunden von Interesse an dem individuellen Kind. Die so aufgebaute Beziehung befriedigt das Bedürfnis nach sozialer Eingebundenheit. Eine sensitive Unterstützung des Kindes bei fachlichen und sozial-emotionalen Problemen fördert das Erleben von Kompetenz und die Möglichkeit der Mitbestimmung erlaubt es den Kindern, sich autonom und selbstbestimmt zu erleben.

Wenngleich in der Forschung zur Qualität pädagogischer Beziehungen bislang häufig die Beziehung der Lehrkraft zum individuellen Kind im Mittelpunkt steht, erscheint aus theoretischer Perspektive auch die gruppenbezogene Beziehung bedeutsam. Prengel (2019) weist ausdrücklich darauf hin, dass im schulischen Kontext neben der individuellen Lehrkraft-Schüler/in-Beziehung auch die Beziehung der Lehrkraft zur Klasse als eigenständige Größe zu berücksichtigen ist. Diese Lehrkraft-Klasse-Beziehung ist parallel zur Lehrkraft-Schüler/in-Beziehung zu fassen als die Art der Wahrnehmung und Ansprache der Schüler/innen als kollektive Gruppe. Die Qualität der Beziehung auf Klassenebene wird bislang vor allem in Studien 
zum Klassenklima thematisiert und dabei in der Regel durch die Aggregierung von Individualinformationen gewonnen (siehe bspw. Ramelow et al. 2015). Dabei bewertet jede/r Schüler/in entweder die Interaktion zwischen der Lehrkraft und ihr/ihm selbst (z. B. ,teacher is strict with me“) oder sie/er beurteilt zusammenfassend das Lehrkraftverhalten gegenüber den Kindern der Klasse (z. B. „teacher is strict“) (den Brok et al. 2006). Die beiden Zugänge können als ,,individuelle“ bzw. ,,generalisierte" Einschätzungen der Beziehungsqualität bezeichnet werden. Auch die Subskalen zur Qualität der Lehrkraft-Schüler/innen-Beziehung in deutschsprachigen Fragebögen zum Klassenklima (z.B. LFSK, Eder und Mayr 2000; von Saldern und Littig 1985) erfassen die Einschätzung jedes einzelnen Kindes, um sie dann auf Gruppenebene zu einer Klimavariable zusammenzufassen.

Die Definition der gruppenbezogenen pädagogischen Beziehung nach Prengel wird unseres Erachtens jedoch durch diese Aggregierung nicht adäquat abgebildet, da hier weiterhin die individuellen Kinder und nicht die Klasse als Ganzes als Beziehungspartner in den Blick genommen werden. Jedoch lässt sich die Beziehungsqualität mit den oben genannten Kriterien (positive Interaktion, Unterstützung, Mitbestimmung) auch auf die Klasse beziehen. Während sich eine positive kindbezogene Interaktion beispielsweise durch einen respektvollen, freundlichen und wertschätzenden Umgang mit dem einzelnen Kind mit seinen Stärken und Schwächen auszeichnet, meint dies auf Gruppenebene die Anerkennung und Wertschätzung der Gruppe in ihrer spezifischen Zusammensetzung. Ein freundlicher Umgangston gegenüber der Klasse als Ganzes oder ein an die Klasse gerichtetes Lob sind Beispiele für Verhaltensweisen, die für eine wertschätzende Lehrkraft-Klasse-Beziehung stehen. Die individuelle Unterstützung bei fachlichen und emotional-sozialen Problemen wird auf Gruppenebene insbesondere durch die Wahrnehmung sozialer Probleme in der Klassengemeinschaft und die angemessene Begleitung bei der Problemlösung gespiegelt. Mitbestimmung kann auf Individualebene durch Autonomieförderung, beispielsweise durch die Möglichkeit Aufgaben, Themen und Lernwege individuell zu wählen sowie die Ermunterung zur individuellen Meinungsäußerung, geschehen. Auf Gruppenebene ist hier die Unterstützung demokratischer Entscheidungsprozesse als Pendant zu sehen.

Bislang gibt es unseres Wissens keine Studien, in denen eine entsprechende Differenzierung der Beziehungsqualität explizit vorgenommen wird. Dabei ist es jedoch gut vorstellbar, dass Lehrkräfte zwischen ihrer individuellen Ansprache des Kindes und der Klasse als Ganzes unterscheiden. Wenn sich ein Kind individuell von der Lehrkraft wenig persönlich angesprochen und wertgeschätzt fühlt, mag eine positive Wahrnehmung des Umgangs mit der ganzen Klasse eventuell kompensatorisch wirken.

\section{Forschungsfragen}

In Abgrenzung und Erweiterung bisheriger Studien differenzieren wir in unserem Beitrag zwischen der durch die Schüler/innen wahrgenommenen Qualität der Beziehung der Lehrkraft zum individuellen Kind und zur Klasse als Ganzes. Wir untersuchen den Einfluss dieser Beziehungsfacetten auf die Schuleinstellung von Kindern 
der Jahrgangsstufen 5 und 6. Mit Blick auf die bisherigen Forschungsbefunde erwarten wir konkret.

a) eine positivere Schuleinstellung für Mädchen, Kinder mit guten Schulleistungen (Noten) und Kinder in Jahrgang 5 im Vergleich zu Kindern des 6. Jahrgangs sowie

b) einen positiven, additiven Effekt beider Facetten der Beziehungsqualität, sodass sowohl die positiv wahrgenommene Beziehungsqualität zum Kind als auch die zur Klasse mit einer positiven Schuleinstellung des Kindes einhergehen.

\section{Methode}

\subsection{Stichprobe}

Insgesamt wurden $N=491$ Schüler/innen aus 22 Gymnasialklassen aus drei Schulen in Nordrhein-Westfalen befragt. Von 14 Kindern lag keine Einverständniserklärung der Eltern vor, sodass diese Fälle aus den Analysen ausgeschlossen wurden. Die Fünft- $(N=254)$ und Sechstklässler/innen $(N=223)$ waren durchschnittlich 10,9 Jahre alt. Die Geschlechterverteilung war ausgeglichen (je 238 Schüler/innen, 1 fehlend). 37,9\% der Kinder wiesen einen Migrationshintergrund auf (operationalisiert über die zu Hause gesprochene Sprache: kein Deutsch oder Deutsch und eine andere Sprache).

\subsection{Instrumente}

Die schriftliche Befragung erfolgte mittels standardisierter und zum Teil neuentwickelter Instrumente.

Die Schuleinstellung wurde operationalisiert in Anlehnung an die Skala Schulunlust von Wieczerkowski et al. (2016). Die Skala besteht aus sechs Items (Beispielitem: Ich bin gerne in der Schule; $\alpha=0,777)$ mit einem vierstufigen Antwortformat $(1=$ trifft nicht $\mathrm{zu}$ bis $4=$ trifft $\mathrm{zu})$.

Zur Erfassung der Qualität der Lehrkraft-Kind-Beziehung (BezQual-Kind) und der Lehrkraft-Klassen-Beziehung (BezQual-Klasse) wurde ein neuentwickeltes Instrument (PBQ-S: Pädagogische Beziehungsqualität aus Schüler/innenperspektive, s. Tab. 3 und 4) genutzt. Das Instrument besteht aus insgesamt 25 Items mit vierstufigem Antwortformat $(1=$ trifft nicht zu bis $4=$ trifft $\mathrm{zu})$, die sich zu zwei Subskalen gruppieren: Die individuelle Beziehungsqualität mit 13 Items (Beispielitem: Lehrkraft A lobt mich häufig; $\alpha=0,809$ ) sowie die gruppenbezogene Beziehungsqualität mit 12 Items (Beispielitem: Lehrkraft A lobt häufig unsere ganze Klasse, z. B. wenn wir im Unterricht gut mitgearbeitet oder leise gearbeitet haben; $\alpha=0,852$ ). Bei den Angaben bezogen sich die Kinder auf ihre Klassenlehrkraft. Die Items bilden die oben dargestellten inhaltlichen Facetten der pädagogischen Beziehungen ab: Die positive Interaktion mit dem individuellen Kind bzw. mit der Klasse als Ganzes, eine sensitive Unterstützung des Kindes bzw. der Klasse als Ganzes insbesondere bei fachlichen und sozial-emotionalen Problemen (auf Klassenebene insbesondere die Unterstützung des Zusammenhalts) sowie die Ermöglichung von Mitbestimmung. 
Die Leistung der Schüler/innen wurde über die von den Schüler/innen berichteten gemittelten Noten in Deutsch und Mathematik auf dem letzten Zeugnis erfasst.

\subsection{Durchführung}

Insgesamt wurden 59 Gymnasien in sechs Städten bzw. Kreisen in Nordrhein-Westfalen zu Beginn des Jahres 2020 zunächst per Email über das Projekt informiert, anschließend telefonisch kontaktiert und um Teilnahme gebeten. Diesem ersten Aufruf folgten lediglich drei Gymnasien. Eine geplante Erweiterung der Stichprobe konnte aufgrund der Corona-Pandemie nicht erfolgen. Die Befragung der Schüler/innen wurde von zwei geschulten Testleiterinnen (studentische Hilfskräfte) unter Nutzung eines Testleitungs-Skripts im Klassenverband im Frühjahr 2020 vor der SchulschlieBung durchgeführt. Zu diesem Zeitpunkt gab es noch keine coronabedingten Einschränkungen im Unterrichtsgeschehen. Der Beginn des zweiten Schulhalbjahres wurde bewusst gewählt, um sicherzustellen, dass sich die Beziehungen zwischen Lehrkraft und Klasse/Schüler/innen bereits in ausreichendem Ausmaß ausgeprägt sind. Die Items wurden laut vorgelesen, die Kinder lasen leise mit und kreuzten ihre entsprechenden Antworten an. Die Lehrkraft war während der Befragung in der Klasse anwesend, saß jedoch in dieser Zeit ausschließlich an ihrem Pult.

\subsection{Auswertungsverfahren}

Die Daten wurden unter Berücksichtigung der Mehrebenenstruktur regressionsanalytisch mit dem Program Mplus (Muthén und Muthén 1998-2017) ausgewertet. Mplus verwendet standardmäßig das Full Information Maximum Likelihood Schätzverfahren zur bestmöglichen Nutzung von Fällen mit fehlenden Werten. Die Anzahl fehlender Werte ist grundsätzlich sehr gering. Bei der Leistungsvariable gibt es 20 fehlende Werte $(=4 \%)$. Bei allen anderen Prädiktoren fehlen nur für jeweils bei 1-2 Fälle gültige Werte $(<0,5 \%)$. Wir nutzen Analysen der Art Type=twolevel zur Definition von Zwei-Ebenen-Modellen. Die Berechnung robuster Standardfehler ermöglicht die reliable Schätzung auch bei Verletzung der Normalverteilungsannahme (Kleinke et al. 2017). Zunächst wurde ein Nullmodell geschätzt, um den Intraklassenkorrelationskoeffizieten (ICC) der abhängigen Variablen und somit die Varianz auf Gruppenebene zu bestimmen. In diesem sogenannten Intercept-Only-Modell sind noch keine Prädiktoren enthalten. Das Modell liefert Schätzungen für die Varianzanteile auf Individual- und Klassenebene (Hox 2010). Im nächsten Schritt (Modell I) wurden aus der Empirie bekannte Prädiktoren als Kontrollvariablen eingebunden. Dies sind auf Individualebene Geschlecht, Migrationshintergrund und Schulleistung sowie auf Klassenebene die Jahrgangsstufe. Im letzten Schritt (Modell II) wurden die beiden z-standardisierten Skalen zur Qualität pädagogischer Beziehungen (BezQual-Kind und BezQual-Klasse) auf Individual- und in aggregierter Form auf Klassenebene hinzugenommen. Die „mittlere“ Beziehungsqualität kann als wichtiges Kontextmerkmal gelten, das Einfluss auf Unterschiede in der mittleren Schuleinstellung zwischen den Klassen nimmt, während das individuelle Beziehungserleben darüber hinaus zu interindividuellen Variationen der Schuleinstellung innerhalb der jeweiligen Klasse beiträgt (Morin et al. 2014). 
Kategoriale Variablen wurden dummy-kodiert mit den Referenzgruppen männlich für Geschlecht, deutsche Familiensprache für Migrationshintergrund und Klasse 5 für Jahrgangsstufe. Alle kontinuierlichen Prädiktoren wurden z-standardisiert.

\section{Ergebnisse}

\subsection{Deskriptive Befunde}

Deskriptive Kennwerte und Korrelationen für die Schulfreude sowie die beiden Beziehungsskalen und Leistung sind in Tab. 1 zusammengefasst.

Die Schuleinstellung korreliert moderat positiv mit beiden Beziehungsvariablen (BezQual-Kind: $r=0,371$, BezQual-Klasse: $r=0,319 ; p<0,001)$ sowie schwach mit der Leistung $(r=-0,103 ; p<0,05)$. Erwartungskonform zeigt sich eine relativ starke Korrelation zwischen den beiden Skalen der Beziehungsqualität $(r=0,699$; $p<0,001)$. Beide Variablen der Beziehungsqualität korrelieren hingegen nur schwach mit der Schulleistung (BezQual-Kind: $r=-0,139 ; p<0,001$; BezQualKlasse: $r=-0,083$; n. s.).

\subsection{Regressionsanalyse}

Die Ergebnisse der verschiedenen Analysen sind in Tab. 2 dargestellt. Das Intercept-Only-Modell weist die Varianzanteile auf Individual- und Klassenebene aus. Die daraus ermittelte Intraklassenkorrelation der abhängigen Variable Schuleinstellung beträgt ICC $=.180$, der Designeffekt DEFF $=4,846$. Es ist also von einer nennenswerten Varianz der mittleren Schuleinstellung über die Schulklassen hinweg auszugehen, sodass eine Berücksichtigung der Zweiebenenstruktur erforderlich ist. Die Konstante $(\mathrm{M}=3,102)$ ist ein deutlicher Hinweis auf eine positive Schuleinstellung in der Stichprobe.

In Modell I zeigt sich, dass Jungen eine negativere Schuleinstellung aufweisen als Mädchen $(\beta=0.159 ; p=0.011)$. Weiterhin sind die Leistung $(\beta=-0.049 ; p=0.010)$ und auf Klassenebene der Jahrgang $(\beta=-0.213 ; p=0.032)$ prädiktiv für die Schuleinstellung. Je besser die Schulleistung, desto positiver die Einstellung gegenüber der Schule. In Jahrgang 6 ist die Einstellung negativer ausgeprägt als in Jahrgang 5. Ein Effekt des Migrationshintergrunds ist statistisch nicht nachweisbar. Modell I erklärt lediglich knapp 3\% der Varianz auf Individualebene, aber 26\% der Varianz auf Klassenebene.

Tab. 1 Skalenkennwerte und Korrelationen

\begin{tabular}{lllllll}
\hline & $\alpha$ & $M(S D)$ & ICC & $(1)$ & $(2)$ & $(3)$ \\
\hline (1) Schuleinstellung & 0,777 & $3,10(0,55)$ & 0,180 & - & - & - \\
(2) BezQual-Kind & 0,809 & $3,26(0,39)$ & 0,223 & $0,371 * * *$ & - & - \\
(3) BezQual-Klasse & 0,852 & $3,32(0,47)$ & 0,293 & $0,319 * * *$ & $0,699 * * *$ & - \\
(4) Leistung & 0,698 & $2,60(0,86)$ & 0,085 & $-0,103 *$ & $-0,139 * * *$ & $-0,083$ \\
\hline
\end{tabular}

$* * * p<0,001, * p<0,05$ 
Tab. 2 Mehrebenenmodell - AV: Schuleinstellung

\begin{tabular}{|c|c|c|c|}
\hline & $\begin{array}{l}\text { Intercept-only-Modell } \\
ß(S E)\end{array}$ & $\begin{array}{l}\text { Modell I } \\
ß(S E)\end{array}$ & $\begin{array}{l}\text { Modell II } \\
ß(S E)\end{array}$ \\
\hline Konstante & $3,102(0,055)^{* * *}$ & $3,216(0,085)^{* * *}$ & $3,188(0,094)^{* * *}$ \\
\hline $\mathrm{ICC}$ & 0,180 & 0,144 & 0,142 \\
\hline \multicolumn{4}{|l|}{ Individualebene } \\
\hline Geschlecht & - & $0,159(0,063)^{*}$ & $0,156(0,054)^{* *}$ \\
\hline Migrationshintergrund & - & $0,103(0,059)$ & $0,085(0,051)$ \\
\hline Leistung & - & $-0,049(0,019)^{*}$ & $-0,043(0,021)^{*}$ \\
\hline BezQual-Kind & - & - & $0,119(0,028)^{* * *}$ \\
\hline BezQual-Klasse & - & - & $0,115(0,042)^{* *}$ \\
\hline \multicolumn{4}{|l|}{ Klassenebene } \\
\hline Jahrgang & - & $-0,213(0,099)^{*}$ & $-0,179(0,094)$ \\
\hline Mean (BezQual-Kind) & - & - & $0,085(0,076)$ \\
\hline $\begin{array}{l}\text { Mean (BezQual-Klas- } \\
\text { se) }\end{array}$ & - & - & $-0,141(0,091)$ \\
\hline \multicolumn{4}{|l|}{ Varianzanteile } \\
\hline Individualebene & $0,246(0,022)$ & $0,238(0,018)$ & $0,204(0,016)$ \\
\hline Klassenebene & $0,054(0,018)$ & $0,040(0,017)$ & $0,034(0,014)$ \\
\hline
\end{tabular}

Geschlecht: $0=$ Junge, Migrationshintergrund: $0=$ ohne, Jahrgang: $0=$ Jahrgang 5

$* * * p<0,001, * * p<0,01, * p<0,05$

Unter Hinzunahme der Beziehungsskalen in Modell II bleibt der Effekt des Geschlechts $(\beta=-0.156 ; p=0.004)$ und der Leistung $(\beta=-0.043 ; p=0.039)$ bestehen. Der Effekt des Jahrgangs $(\beta=-0.179 ; p=0.055)$ ist nur noch marginal. Hypothesenkonform zeigt sich, dass beide Beziehungsskalen auf Individualebene prädiktiv für die Schulfreude sind (BezQual-Kind: $\beta=-0.119 ; p<0.001$ ); BezQual-Klasse: $\beta=0.115 ; p=0.006)$. Auf Level 2 ist ein Effekt der aggregierten pädagogischen Beziehungen statistisch nicht abzusichern. Modell II erklärt im Vergleich zum Nullmodell gut $17 \%$ der Varianz auf Individual- und $37 \%$ der Varianz auf Klassenebene.

\section{Diskussion}

\subsection{Zentrale Befunde}

Die Ergebnisse bestätigen Befunde bisheriger Studien, nach denen männliche und ältere Schüler/innen im Vergleich zu ihren weiblichen und jüngeren Mitschüler/ innen eine negativere Schuleinstellung aufweisen. Ein Effekt des Migrationshintergrundes war, wie auch in anderen Studien, nicht nachweisbar (z.B. Harazd und Schürer 2006; Kohl et al. 2011; Okun et al. 1990). Schulleistung - erfasst über die Noten in Deutsch und Mathematik - und Schuleinstellung korrelieren nur gering. Dies verwundert zunächst, da gute Noten mit hohem Kompetenzerleben und positiven Leistungsemotionen einhergehen (Pekrun et al. 2017). So finden sich in der Schulforschung wiederholt positive Korrelationen zwischen Wohlbefinden und Schulleistungen (Gutman und Vorhaus 2012; Hascher 2004; Hascher und Hagenauer 
2011; Noble et al. 2008). Allerdings sind auch dort die Zusammenhänge eher gering und es liegen auch Studien vor, die keinen Zusammenhang zur Leistung zeigen (Lee 2016). Aus pädagogischer Sicht ist der Befund durchaus als positiv zu werten, besagt er doch, dass Schulfreude im Sinne einer positiven affektiven Schuleinstellung auch unabhängig von der Schulleistung erreicht werden kann. Zu bedenken sind jedoch auch Verzerrungen aufgrund der Selbstauskunft der Noten durch die Schüler/ innen. Um diese zukünftig auszuschließen, wäre eine Abfrage der Noten durch die Lehrkräfte denkbar.

Der Fokus der Studie lag auf der Rolle der durch das Kind wahrgenommenen Beziehungsqualität, wobei neben der Beziehung der Lehrkraft zum individuellen Kind auch die Beziehung zur Klasse als Ganzes betrachtet wurde. Beide Facetten der Beziehungsqualität erwiesen sich als bedeutsame Prädiktoren der Schuleinstellung. Kinder, die sich von ihrer Klassenlehrkraft anerkannt sowie fachlich und emotional unterstützt fühlen und die erleben, dass ihre Meinung zählt, berichten auch, dass sie gerne zur Schule gehen und diesen Ort und die Lernaktivitäten mit positiven Gefühlen assoziieren. Darüber hinaus liefert auch eine positiv wahrgenommene Beziehung zwischen Lehrkraft und Klasse einen wichtigen Beitrag für das Erleben von Schulfreude. So erleben die Kinder eine höhere Schulfreude, die wahrnehmen, dass die Lehrkraft die Klasse mag, auf das Zusammenleben in der Klasse achtet und bei sozialen Problemen aktiv unterstützt sowie demokratische Prozesse und Mitbestimmung befürwortet. Wenngleich die beiden Facetten der Beziehungsqualität hoch miteinander korrelieren, sprechen die regressionsanalytischen Ergebnisse doch dafür, sie als separate Konstrukte weiter im Blick zu halten. Bisherige Klimainstrumente nehmen eine solche Differenzierung nicht vor. Das von uns eingesetzte Instrument, der PBQ-S, stellt eine erste Möglichkeit dar, sowohl die Beziehung der Lehrkraft zum individuellen Kind als auch die gruppenbezogene Beziehung zu erfassen. Im Gegensatz zur Individualebene war auf Klassenebene kein Effekt der Beziehungsqualität auf die Schuleinstellung nachweisbar, sodass zunächst davon auszugehen ist, dass sich die Beziehungsqualität primär im individuellen Erleben niederschlägt.

\subsection{Limitationen und Ausblick}

Bei der Interpretation der Befunde ist zu berücksichtigen, dass bisher lediglich Daten aus Gymnasien vorliegen und die Stichprobengröße noch recht gering ist. Von den knapp 60 angefragten Schulen haben lediglich drei Schulen an unserer Befragung teilgenommen. Somit ist davon auszugehen, dass es sich um eine PositivSelektion von Schulen handelt. Gerade bei Zusammenhangsanalysen sind jedoch geringe Variationen in Prädiktoren oder Kriterium problematisch. Eine Erweiterung der Stichprobe, insbesondere auch um Schulen anderer Schulformen, deren Schülerschaft im Mittel weniger positiv gegenüber Schule eingestellt ist (van Ophuysen 2008), ist daher wünschenswert.

Unabhängig von der Problematik zur Aussagekraft der Ergebnisse stellt sich die Frage, warum Schulen bzw. Lehrkräfte wenig Bereitschaft zeigten, mit ihren Schüler/innen an der kurzen Befragung teilzunehmen. In telefonischen Gesprächen wurde von den Schulleitungen durchaus eine Bereitschaft zur Teilnahme an wissenschaftlichen Studien signalisiert. Jedoch wurde die Thematik der Studie als problematisch 
eingestuft. Lehrkräfte scheinen einer schülerseitigen Beurteilung ihrer Beziehungen zu den Schüler/innen bzw. zur Klasse selbst bei Zusicherung absoluter Vertraulichkeit der Daten gegenüber Dritten ausgesprochen skeptisch gegenüberzustehen. Um eine höhere Beteiligung zu erreichen, erscheint es sinnvoll, die Beweggründe von Lehrkräften zu eruieren, um auf eventuelle Befürchtungen reagieren und damit die Teilnahmebereitschaft erhöhen zu können.

Das Modell erklärt sowohl auf Individual- als auch auf Gruppenebene etwa ein Fünftel der Varianz. Es erscheint gewinnbringend, in zukünftigen Studien auch die Beziehung unter den Schüler/innen als Prädiktor zu berücksichtigen. Perdue et al. (2009) zeigen, dass die Einbindung in Freundschaften und das Vorhandensein positiver Sozialbeziehungen eine wesentliche Motivation für die Schüler/innen darstellt, Schule zu besuchen. Auch andere Autor/innen verweisen auf den Zusammenhang zwischen der Qualität der Beziehungen unter den Schüler/innen und der Schuleinstellung (Gysin 2017; Hascher und Baillod 2009; Hascher und Lobsang 2004).

$\mathrm{Zu}$ bedenken ist weiterhin, dass das schulische Wohlbefinden als mehrdimensionales Konstrukt zu verstehen ist, das nach dem Modell von Hascher (2004) neben den habituellen auch aktuelle Facetten beinhaltet. In unseren Analysen wurde mit der Einstellung zur Schule eine habituelle, stabile Facette als abhängige Variable gewählt. In weiteren Untersuchungen könnten zusätzlich Merkmale des aktuellen Wohlbefindens im konkreten Unterricht bei derjenigen Lehrkraft fokussiert werden, für die die Schüler/innen auch die Beziehungsqualität beurteilen. Ebenfalls erscheint es spannend, wenn jedes Kind für mehrere Lehrkräfte die Beziehungsqualität und das aktuelle Wohlbefinden im Unterricht einschätzt, um den Einfluss der Beziehungsqualität besser von Merkmalen des Kindes trennen zu können. Weiterhin könnte in zukünftigen Studien abgefragt werden, wie lange und mit wie vielen Stunden die Schüler/innen bereits von der Lehrkraft unterrichtet werden, um dies als Kontrollvariablen mit in die Analysen aufzunehmen.

Ebenso wie das Wohlbefinden kann auch die pädagogische Beziehungsqualität differenzierter betrachtet werden. Das von uns entwickelte Erhebungsinstrument (PBQ-S) erfasst die Qualität der Beziehung zum Kind sowie zur Klasse jeweils über drei Subskalen (,positive Interaktion“, „Unterstützung“, „Mitbestimmung“), die aufgrund der geringen Stichprobengröße zu einem Gesamtwert zusammengefasst wurden. Es erscheint plausibel anzunehmen, dass die Subskalen je mit spezifischen Komponenten des schulischen Wohlbefindens zusammenhängen (z. B. (soziale) Unterstützung der Klasse als Ganzes $\leftrightarrow$ Abwesenheit sozialer Probleme; (fachliche) Unterstützung des Kindes $\leftrightarrow$ Abwesenheit schulischer Sorgen). Auch dies kann in weiteren Studien betrachtet werden.

\subsection{Implikationen für die schulische Praxis}

Mit Blick auf die schulische Praxis ergeben sich aus den bisherigen Ergebnissen bereits jetzt wichtige Implikationen. Dass Kinder eine positive Einstellung gegenüber der Schule entwickeln, ist eine inzwischen allgemein anerkannte Zielsetzung der Institution Schule (Hagenauer 2011). Die vorliegende Studie verdeutlicht einerseits einen bereits bekannten Befund: Der respektvolle und wertschätzende Umgang mit dem individuellen Kind, der fürsorgliche Blick auf seine fachlichen und sozialen 
Bedürfnisse und das Ermöglichen von Mitbestimmung - oder, zusammenfassend gesagt, eine vom Kind positiv erlebte Pädagogische Beziehung - unterstützt den Aufbau dieser positiven schulbezogenen Haltung. Lehrkräfte sind daher dazu angehalten, ihre Beziehung zum individuellen Kind so zu gestalten, dass sie die von Deci und Ryan in der Selbstbestimmungstheorie formulierten Grundbedürfnisse der Schüler/innen adressieren: Ermöglichung des Bedürfnisses nach sozialer Eingebundenheit durch positive Interaktion mit dem Kind, des Kompetenzerlebens durch entsprechende Unterstützung sowie von Autonomie durch die Möglichkeit von Mitbestimmung.

Neben dem Blick auf die Gestaltung der Beziehung zum individuellen Kind, sollten sich Lehrkräfte jedoch auch bewusstmachen, dass sie häufig die Klasse als Ganzes adressieren, und so eine Beziehung zu der Klasse etablieren. Ein positives Merkmal dieser Beziehung stellt beispielsweise die aufmerksame Wahrnehmung der sozialen Strukturen in der Klasse dar, um bei eventuellen Problemen den sozialen Zusammenhalt und das gemeinsame Lernen in der Klasse mit entsprechenden Unterstützungsangeboten zu fördern. Auch auf Klassenebene gilt es Mitbestimmung der Schülerschaft als wichtiges Beziehungsmerkmal zu ermöglichen (Prengel 2019). Durch das Einbinden der Klasse in Entscheidungsprozesse und die Ermöglichung von Elementen gelebter Demokratie im Klassenraum kann die Lehrkraft die Beziehung zur Gruppe gestalten und verbessern (z. B. Klassenrat). Schließlich ist mit Blick auf eine positive Interaktion gegenüber der Klasse als Ganzes die Anerkennung der Gruppe in ihrer spezifischen Zusammensetzung und damit insbesondere auch in ihrer Vielfalt und Heterogenität bedeutsam. Vielfalt als Chance zu verstehen, bedarf jedoch ggf. Einstellungsveränderungen, die nicht immer leicht zu erreichen sind. Hier gilt es in der Lehrkräfteausbildung zu sensibilisieren. Wenn es der Lehrkraft gelingt, der Klassengemeinschaft in ihrer Diversität Wertschätzung entgegen zu bringen und dies für die Kinder deutlich erkennbar ist, verstärkt auch dies - so unsere Daten - die Chancen auf eine positive schulbezogene Einstellung bei den Schüler/innen. 


\section{Anhang}

\section{QPB-S}

Tab. 3 Subskala Qualität der Lehrkraft-Kind-Beziehung (BezQual-Kind)

\begin{tabular}{|c|c|c|c|c|c|}
\hline & Klassenlehrkraft A ... & $\begin{array}{l}\text { Trifft } \\
\mathrm{zu}\end{array}$ & $\begin{array}{l}\text { Trifft } \\
\text { eher zu }\end{array}$ & $\begin{array}{l}\text { Trifft } \\
\text { eher } \\
\text { nicht zu }\end{array}$ & $\begin{array}{l}\text { Trifft } \\
\text { nicht zu }\end{array}$ \\
\hline AI01 & ... ist mir gegenüber freundlich & $\square$ & $\square$ & $\square$ & $\square$ \\
\hline $\mathrm{AI} 02$ & ... lobt mich häufig & $\square$ & $\square$ & $\square$ & $\square$ \\
\hline AI03 & ... zeigt mir, dass sie mich mag & $\square$ & $\square$ & $\square$ & $\square$ \\
\hline AI04 & ... interessiert sich dafür, wie es mir geht & $\square$ & $\square$ & $\square$ & $\square$ \\
\hline AI05 & $\begin{array}{l}\text {... würde mir helfen, wenn ich mal Proble- } \\
\text { me oder Streit mit anderen Kindern habe }\end{array}$ & $\square$ & $\square$ & $\square$ & $\square$ \\
\hline AI06 & $\begin{array}{l}\text {... würde mir helfen, wenn im Unterricht } \\
\text { etwas mal nicht so gut klappt }\end{array}$ & $\square$ & $\square$ & $\square$ & $\square$ \\
\hline AI07 & ... nimmt mich dran, wenn ich mich melde & $\square$ & $\square$ & $\square$ & $\square$ \\
\hline AI08 & $\begin{array}{l}\text {... weiß was ich beim Arbeiten in der Schule } \\
\text { gut kann und was mir noch schwerfällt } \\
\text { (z.B. sorgfältig sein, mich konzentrieren, } \\
\text { mit anderen im Team zusammenarbeiten) }\end{array}$ & $\square$ & $\square$ & $\square$ & $\square$ \\
\hline AI09 & $\begin{array}{l}\text {.. weiß welche Aufgaben für mich genau } \\
\text { richtig sind - also weder zu schwierig noch } \\
\text { zu leicht }\end{array}$ & $\square$ & $\square$ & $\square$ & $\square$ \\
\hline AI10 & $\begin{array}{l}\text {... weiß in welche Klasse ich gehöre, auch } \\
\text { wenn sie mich mal außerhalb des Klassen- } \\
\text { raums trifft }\end{array}$ & $\square$ & $\square$ & $\square$ & $\square$ \\
\hline AI11 & $\begin{array}{l}\ldots \text { weiß, mit wem ich mich in der Klasse gut } \\
\text { verstehe, und mit wem nicht so gut }\end{array}$ & $\square$ & $\square$ & $\square$ & $\square$ \\
\hline AI12 & $\begin{array}{l}\text {... hört mir immer aufmerksam zu, wenn ich } \\
\text { ihr etwas erzähle oder meine Meinung sage }\end{array}$ & $\square$ & $\square$ & $\square$ & $\square$ \\
\hline AI13 & $\begin{array}{l}\text {... lässt mich ich im Unterricht oder bei den } \\
\text { Hausaufgaben auch mal selbst entscheiden } \\
\text { was ich machen möchte }\end{array}$ & $\square$ & $\square$ & $\square$ & $\square$ \\
\hline
\end{tabular}


Tab. 4 Subskala Qualität der Lehrkraft-Klassen-Beziehung (BezQual-Klasse)

\begin{tabular}{|c|c|c|c|c|c|}
\hline & Klassenlehrkraft A ... & $\begin{array}{l}\text { Trifft } \\
\text { zu }\end{array}$ & $\begin{array}{l}\text { Trifft } \\
\text { eher zu }\end{array}$ & $\begin{array}{l}\text { Trifft } \\
\text { eher nicht } \\
\text { zu }\end{array}$ & $\begin{array}{l}\text { Trifft } \\
\text { nicht zu }\end{array}$ \\
\hline AG01 & $\begin{array}{l}\text {... lobt häufig unsere ganze Klasse (z.B. } \\
\text { wenn wir im Unterricht gut mitgearbeitet } \\
\text { oder leise gearbeitet haben) }\end{array}$ & $\square$ & $\square$ & $\square$ & $\square$ \\
\hline AG02 & $\begin{array}{l}\text {... sagt uns, dass sie gerne in unserer Klasse } \\
\text { unterrichtet }\end{array}$ & $\square$ & $\square$ & $\square$ & $\square$ \\
\hline AG03 & $\begin{array}{l}\text {... findet es gut, dass wir in unserer Klasse } \\
\text { alle unterschiedlich sind (z. B. in unseren } \\
\text { Meinungen, Leistungen, Fähigkeiten und } \\
\text { in unserem Verhalten) }\end{array}$ & $\square$ & $\square$ & $\square$ & $\square$ \\
\hline AG04 & $\begin{array}{l}\text {... achtet darauf, dass in unserer Klasse } \\
\text { niemand extra ausgegrenzt oder gemobbt } \\
\text { wird }\end{array}$ & $\square$ & $\square$ & $\square$ & $\square$ \\
\hline AG05 & $\begin{array}{l}\text {... achtet darauf, dass alle Kinder bei Grup- } \\
\text { penarbeiten einbezogen werden und jeder } \\
\text { mitarbeiten kann }\end{array}$ & $\square$ & $\square$ & $\square$ & $\square$ \\
\hline AG06 & $\begin{array}{l}\text {... achtet darauf, dass alle Kinder in den } \\
\text { Pausen einbezogen werden und jeder mit- } \\
\text { machen kann }\end{array}$ & $\square$ & $\square$ & $\square$ & $\square$ \\
\hline AG07 & $\begin{array}{l}\text {... achtet darauf, dass in unserer Klasse } \\
\text { niemand alleine bleibt und nicht beachtet } \\
\text { wird }\end{array}$ & $\square$ & $\square$ & $\square$ & $\square$ \\
\hline AG08 & $\begin{array}{l}\text {... achtet darauf, dass wir in unserer Klasse } \\
\text { alle freundlich miteinander umgehen }\end{array}$ & $\square$ & $\square$ & $\square$ & $\square$ \\
\hline AG09 & $\begin{array}{l}\ldots \text { achtet darauf, dass wir uns in unserer } \\
\text { Klasse gegenseitig helfen }\end{array}$ & $\square$ & $\square$ & $\square$ & $\square$ \\
\hline AG10 & $\begin{array}{l}\text {... achtet darauf, dass wir als Klasse alle gut } \\
\text { zusammenhalten }\end{array}$ & $\square$ & $\square$ & $\square$ & $\square$ \\
\hline AG11 & $\begin{array}{l}\text {... fragt uns als Klasse bei vielen Dingen } \\
\text { nach unserer Meinung (z. B. beim Klären } \\
\text { von Streitigkeiten) }\end{array}$ & $\square$ & $\square$ & $\square$ & $\square$ \\
\hline AG12 & $\begin{array}{l}\text {... lässt uns als Klasse bei vielen Entschei- } \\
\text { dungen mitbestimmen (z.B. zu Ausflügen, } \\
\text { zur Klassenraumgestaltung, zur Sitzord- } \\
\text { nung) }\end{array}$ & $\square$ & $\square$ & $\square$ & $\square$ \\
\hline
\end{tabular}

Funding Open Access funding enabled and organized by Projekt DEAL.

Open Access Dieser Artikel wird unter der Creative Commons Namensnennung 4.0 International Lizenz veröffentlicht, welche die Nutzung, Vervielfältigung, Bearbeitung, Verbreitung und Wiedergabe in jeglichem Medium und Format erlaubt, sofern Sie den/die ursprünglichen Autor(en) und die Quelle ordnungsgemäß nennen, einen Link zur Creative Commons Lizenz beifügen und angeben, ob Änderungen vorgenommen wurden.

Die in diesem Artikel enthaltenen Bilder und sonstiges Drittmaterial unterliegen ebenfalls der genannten Creative Commons Lizenz, sofern sich aus der Abbildungslegende nichts anderes ergibt. Sofern das betreffende Material nicht unter der genannten Creative Commons Lizenz steht und die betreffende Handlung nicht nach gesetzlichen Vorschriften erlaubt ist, ist für die oben aufgeführten Weiterverwendungen des Materials die Einwilligung des jeweiligen Rechteinhabers einzuholen. 
Weitere Details zur Lizenz entnehmen Sie bitte der Lizenzinformation auf http://creativecommons.org/ licenses/by/4.0/deed.de.

\section{Literatur}

Aldridge, J. M., Fraser, B. J., Fozdar, F., Ala'i, K., Earnest, J., \& Afari, E. (2016). Students' perceptions of school climate as determinants of wellbeing, resilience and identity. Improving Schools, 19(1), 5-26. https://doi.org/10.1177/1365480215612616.

Becker, P. (1994). Wohlbefinden. In A. Abele \& P. Becker (Hrsg.), Wohlbefinden: Theorie - Empirie Diagnostik 2. Aufl. Juventa-Materialien. (S. 13-49). Weinheim, München: Juventa.

Beisenherz, G. (2005). Wie wohl fühlst Du Dich? Kindliche Persönlichkeit und Umwelt als Quelle von Wohlbefinden und Unwohlsein bei Grundschülern. In C. Alt (Hrsg.), Aufwachsen in Familien 1. Aufl. Kinderleben - Aufwachsen zwischen Familie, Freunden und Institutionen, (Bd. 1, S. 157-186). Wiesbaden: VS.

den Brok, P., Brekelmans, M., \& Wubbels, T. (2006). Multilevel issues in research using students' perceptions of learning environments: the case of the questionnaire on teacher interaction. Learning Environments Research, 9(3), 199-213. https://doi.org/10.1007/s10984-006-9013-9.

Czerwenka, K., Nölle, K., Pause, G., Schlotthaus, W., Schmidt, H.-J., \& Tessloff, J. (1990). Schülerurteile über die Schule: Bericht über eine internationale Untersuchung. Europäische Hochschulschriften Reihe 11, Bd. 419. Frankfurt: Peter Lang.

Deci, E.L., \& Ryan, R.M. (2000). The "what" and "why" of goal pursuits: Human needs and the self-determination of behavior. Psychological Inquiry, 11(4), 227-268. https://doi.org/10.1207/ S15327965PLI1104_01.

Diener, E., Suh, E. M., Lucas, R.E., \& Smith, H.L. (1999). Subjective well-being: three decades of progress. Psychological Bulletin, 125(2), 276-302. https://doi.org/10.1037/0033-2909.125.2.276.

Eder, F. (1995). Das Befinden von Schülerinnen und Schülern in den öffentlichen Schulen. In F. Eder (Hrsg.), Das Befinden von Kindern und Jugendlichen in der Schule: Forschungsbericht im Auftrag des $B M U K$. Bildungsforschung des Bundesministeriums für Unterricht und Kulturelle Angelegenheiten, (Bd. 8, S. 24-168). Innsbruck: Studienverlag.

Eder, F. (2002). Unterrichtsklima und Unterrichtsqualität. Unterrichtswissenschaft, 30(2), 213-229.

Eder, F. (2007). Das Befinden von Kindern und Jugendlichen in der österreichischen Schule: Befragung 2005. Bildungsforschung des Bundesministeriums für Bildung, Wissenschaft und Kultur, Bd. 20. Wien: Studienverlag.

Eder, F., \& Mayr, J. (2000). Linzer Fragebogen zum Schul- und Klassenklima für die 4.-8. Klassenstufe (LFSK 4-8). Göttingen: Hogrefe.

Fend, H. (1997). Der Umgang mit Schule in der Adoleszenz: Aufbau und Verlust von Lernmotivation, Selbstachtung und Empathie. Entwicklungspsychologie der Adoleszenz in der Moderne, Bd. 4. Bern: Huber.

Fend, F., \& Sandmeier, A. (2004). Wohlbefinden in der Schule: „Wellness“ oder Indiz für gelungene Pädagogik? In T. Hascher (Hrsg.), Schule positiv erleben: Ergebnisse und Erkenntnisse zum Wohlbefinden von Schülerinnen und Schülern. Schulpädagogik - Fachdidaktik - Lehrerbildung, (Bd. 10, S. 161-184). : Haupt.

Gläser-Zikuda, M., \& Fuß, S. (2004). Wohlbefinden von Schülerinnen und Schülern im Unterricht. In T. Hascher (Hrsg.), Schule positiv erleben: Ergebnisse und Erkenntnisse zum Wohlbefinden von Schülerinnen und Schülern. Schulpädagogik - Fachdidaktik - Lehrerbildung, (Bd. 10, S. 27-48). Bern: Haupt.

Grewe, N. (2017). Soziale Interaktion und Klassenklima. In M. K. W. Schweer (Hrsg.), Lehrer-SchülerInteraktion (S. 547-560). Wiesbaden: Springer. https://doi.org/10.1007/978-3-658-15083-9_24.

Gutman, L. M., \& Vorhaus, J. (2012). The impact of pupil behaviour and wellbeing on educational outcomes. Research report (DFE-RR253). Institute of Education, University of London. Childhood Wellbeing Research Centre, Department for Education. https://www.basw.co.uk/system/files/resources/ basw_22531-3_0.pdf. Zugegriffen: 21. Apr. 2021.

Gysin, S. (2017). Subjektives Wohlbefinden von Schülerinnen und Schülern. Weinheim, Basel: Beltz Juventa.

Hagenauer, G. (2011). Lernfreude in der Schule. Pädagogische Psychologie und Entwicklungspsychologie, Bd. 80. Münster: Waxmann. 
Hamre, B. K., Pianta, R.C., Downer, J. T., DeCoster, J., Mashburn, A. J., Jones, S. M., Brown, J.L., Cappella, E., Atkins, M., Rivers, S. E., Brackett, M. A., \& Hamagami, A. (2013). Teaching through interactions. The Elementary School Journal, 113(4), 461-487. https://doi.org/10.1086/669616.

Harazd, B., \& Schürer, S. (2006). Veränderung der Schulfreude von der Grundschule zur weiterführenden Schule. In A. Schründer-Lenzen (Hrsg.), Risikofaktoren kindlicher Entwicklung: Migration, Leistungsangst und Schulübergang (1. Aufl. S. 208-222). Wiesbaden: VS. https://doi.org/10.1007/9783-531-90075-9_11.

Hascher, T. (2004). Wohlbefinden in der Schule (1. Aufl.). Pädagogische Psychologie und Entwicklungspsychologie, Bd. 40. Münster: Waxmann.

Hascher, T., \& Baillod, J. (2009). Soziale Integration in der Schulklasse als Prädiktor für Wohlbefinden. In T. Hascher (Hrsg.), Schule positiv erleben. Ergebnisse und Erkenntnisse zum Wohlbefinden von Schülerinnen und Schülern (S. 133-158). Bern: Haupt. https://doi.org/10.7892/BORIS.52840.

Hascher, T., \& Edlinger, H. (2009). Positive Emotionen und Wohlbefinden in der Schule: Ein Überblick über Forschungszugänge und Erkenntnisse. Psychologie in Erziehung und Unterricht, 56(2), 105-122. https://doi.org/10.7892/BORIS.52327.

Hascher, T., \& Hagenauer, G. (2011). Schulisches Wohlbefinden im Jugendalter: Verläufe und Einflussfaktoren. In A. Ittel, H. Merkens \& L. Stecher (Hrsg.), Jahrbuch Jugendforschung (10. Aufl. S. 15-45). Wiesbaden: VS. https://doi.org/10.1007/978-3-531-93116-6_1.

Hascher, T., \& Lobsang, K. (2004). Das Wohlbefinden von SchülerInnen - Faktoren, die es stärken und solche, die es schwächen. In T. Hascher (Hrsg.), Schule positiv erleben: Ergebnisse und Erkenntnisse zum Wohlbefinden von Schülerinnen und Schülern. Schulpädagogik - Fachdidaktik - Lehrerbildung, (Bd. 10, S. 203-228). Bern: Haupt.

Hascher, T., Hagenauer, G., \& Schaffer, A. (2011). Wohlbefinden in der Grundschule. Erziehung \& Unterricht, 161(3/4), 381-392. https://doi.org/10.7892/BORIS.52312.

Hascher, T., Morinaj, J., \& Waber, J. (2018). Schulisches Wohlbefinden: Eine Einführung in Konzept und Forschungsstand. In K. Rathmann \& K. Hurrelmann (Hrsg.), Leistung und Wohlbefinden in der Schule: Herausforderung Inklusion (1. Aufl. S. 66-82). Weinheim Basel: Beltz Juventa.

Helmke, A. (1993). Die Entwicklung der Lernfreude vom Kindergarten bis zur 5. Klassenstufe. Zeitschrift für Pädagogische Psychologie, 7, 77-86.

Hox, J. J. (2010). Multilevel analysis: techniques and applications. London: Routledge.

Kirsch, B., \& Hansen, I. (2002). Schulunlust - ein mögliches Initialsymptom für Schulvermeidung - untersucht an Schülern vor dem Übergang von der 6. zur 7. Klasse. Heilpädagogische Forschung, 28, 58-68.

Kleinke, K., Schlüter, E., \& Christ, O. (2017). Strukturgleichungsmodelle mit Mplus: Eine praktische Einführung (2. Aufl.). Berlin, Boston: De Gruyter Oldenbourg. https://doi.org/10.1515/9783486989458.

Kohl, K., Striegler, K., Peters, K., \& Leyendecker, B. (2011). Positive Schuleinstellung, Lernfreude und respektvolle Schüler-Lehrer-Beziehung: Die Situation von Kindern aus zugewanderten Familien in der Grundschule. In A. Ittel, H. Merkens \& L. Stecher (Hrsg.), Jahrbuch Jugendforschung (10. Aufl. S. 46-73). Wiesbaden: VS. https://doi.org/10.1007/978-3-531-93116-6_2.

Külker, A., Dorniak, M., Geist, S., Kullmann, H., Lutter, N., Lütje-Klose, B., \& Siepmann, C. (2017). Schulisches Wohlbefinden als Qualitätsmerkmal inklusiver Schulen: Unterrichtsentwicklung im Rahmen eines Lehrer-Forscher-Projekts an der Laborschule Bielefeld. In A. Textor, S. Grüter, I. Schiermeyer-Reichl \& B. Streese (Hrsg.), Leistung Inklusive? Inklusion in der Leistungsgesellschaft (Bd. 2, S. 48-59). Bad Heilbrunn: Julius Klinkhardt.

Lee, J. (2016). Attitude toward school does not predict academic achievement. Learning and Individual Differences, 52(1), 1-9. https://doi.org/10.1016/j.lindif.2016.09.009.

Mayr, T., \& Ulich, M. (2002). Wohlbefinden im späten Kindes- und frühen Jugendalter: Wie erleben Kinder/Jugendliche Familie, Freunde und Schule? In LBS-Initiative Junge Familie (Hrsg.), Kindheit 2001 Das LBS-Kinderbarometer: Was Kinder wünschen, hoffen und befürchten (S. 45-69). Opladen: Leske + Budrich. https://doi.org/10.1007/978-3-322-92258-8_3.

Morin, A. J.S., Marsh, H. W., Nagengast, B., \& Scalas, L.F. (2014). Doubly latent multilevel analyses of classroom climate: an illustration. The Journal of Experimental Education, 82(2), 143-167. https:// doi.org/10.1080/00220973.2013.769412.

Muthén, L., \& Muthén, B. (2017). Mplus user's guide (8. Aufl.). http://www.statmodel.com/download/ usersguide/MplusUserGuideVer_8.pdf. Zugegriffen: 21. Apr. 2021.

Noble, T., McGrath, H., Roffey, S., \& Rowling, L. (2008). Scoping study into approaches to student wellbeing: Final report (PRN 18219). Brisbane. https://docs.education.gov.au/documents/scoping-studyapproaches-student-wellbeing-final-report. Zugegriffen: 21. Apr. 2021. 
Okun, M. A., Braver, M. W., \& Weir, R. M. (1990). Grade level differences in school satisfaction. Social Indicators Research, 22(4), 419-427. https://doi.org/10.1007/BF00303835.

van Ophuysen, S. (2008). Zur Veränderung der Schulfreude von Klasse 4 bis 7: Eine Längsschnittanalyse schulformspezifischer Effekte von Ferien und Grundschulübergang. Zeitschrift für Pädagogische Psychologie, 22, 293-306. https://doi.org/10.1024/1010-0652.22.34.293.

Pekrun, R., Lichtenfeld, S., Marsh, H.W., Murayama, K., \& Goetz, T. (2017). Achievement emotions and academic performance: longitudinal models of reciprocal effects. Child Development, 88(5), 1653-1670. https://doi.org/10.1111/cdev.12704.

Perdue, N.H., Manzeske, D.P., \& Estell, D. B. (2009). Early predictors of school engagement: exploring the role of peer relationships. Psychology in the Schools, 46(10), 1084-1097. https://doi.org/10.1002/ pits.20446.

van Petegem, K., Aelterman, A., van Keer, H., \& Rosseel, Y. (2008). The influence of student characteristics and interpersonal teacher behaviour in the classroom on student's wellbeing. Social Indicators Research, 85(2), 279-291. https://doi.org/10.1007/s11205-007-9093-7.

Prengel, A. (2019). Pädagogische Beziehungen zwischen Anerkennung, Verletzung und Ambivalenz (2. Aufl.). Opladen: Barbara Budrich.

Primas, S. (2014). Depressive Befindlichkeit und gesundheitsbezogene Lebensqualität im Kindesalter: Eine empirische Studie an Schülerinnen und Schülern der 3. bis 6. Schulstufe. Saarbrücken: Akademikerverlag.

Pupeter, M., \& Hurrelmann, K. (2013). Die Schule: Als Erfahrungsraum immer wichtiger. In S. Andresen \& K. Hurrelmann (Hrsg.), Wie gerecht ist unsere Welt? 3. World Vision Kinderstudie. Kinder in Deutschland, (Bd. 3, S. 111-134). Weinheim, Basel: Beltz.

Ramelow, D., Currie, D., \& Felder-Puig, R. (2015). The assessment of school climate. Journal of Psychoeducational Assessment, 33(8), 731-743. https://doi.org/10.1177/0734282915584852.

Rauer, W., \& Schuck, K. D. (2003). FEESS 3-4. Fragebogen zur Erfassung emotionaler und sozialer Schulerfahrungen von Grundschulkindern dritter und vierter Klassen. Göttingen: Beltz.

Ruzek, E.A., Hafen, C.A., Allen, J.P., Gregory, A., Mikami, A. Y., \& Pianta, R.C. (2016). How teacher emotional support motivates students: the mediating roles of perceived peer relatedness, autonomy support, and competence. Learning and Instruction, 42, 95-103. https://doi.org/10.1016/j. learninstruc.2016.01.004.

von Saldern, M. (1987). Sozialklima von Schulklassen: Überlegungen und mehrebenenanalytische Untersuchung zur subjektiven Wahrnehmung von Lernumwelten. Dissertation. Frankfurt am Main: GoetheUniversität Frankfurt am Main.

von Saldern, M., \& Littig, K. E. (1985). Die Konstruktion der Landauer Skalen zum Sozialklima (Lasso). Zeitschrift für Entwicklungspsychologie und Pädagogische Psychologie, 17, 138-149.

Schwab, S., Rossmann, P., Tanzer, N., Hagn, J., Oitzinger, S., Thurner, V., \& Wimberger, T. (2015). Schulisches Wohlbefinden von SchülerInnen mit und ohne sonderpädagogischen Förderbedarf: Integrations- und Regelklassen im Vergleich. Zeitschrift für Kinder- und Jugendpsychiatrie und Psychotherapie. https://doi.org/10.1024/1422-4917/a000363.

Serke, B. (2019). Schulisches Wohlbefinden in inklusiven und exklusiven Schulmodellen: Eine empirische Studie zur Wahrnehmung und Förderung des schulischen Wohlbefindens von Kindern mit sonderpädagogischem Förderbedarf Lernen. Perspektiven sonderpädagogischer Forschung. Bielefeld: Julius Klinkhardt.

Stanat, P., \& Christensen, G. (2006). Schulerfolg von Jugendlichen mit Migrationshintergrund im internationalen Vergleich: Eine Analyse von Voraussetzungen und Erträgen schulischen Lernens im Rahmen von PISA 2003. Berlin: Bundesministerium für Bildung und Forschung (BMBF).

Veith, H. (2016). Gute Schulen investieren in ihre Kultur: Die Perspektive der Wissenschaft. In S.-I. Beutel, K. Höhmann, H. A. Pant \& M. Schratz (Hrsg.), Handbuch gute Schule: Sechs Qualitätsbereiche für eine zukunftsweisende Praxis (2. Aufl. S. 116-129). Seelze: Klett.

Verkuyten, M., \& Thijs, J. (2002). School satisfaction of elementary school children: the role of performance, peer relations, ethnicity and gender. Social Indicators Research, 59(2), 203-228. https://doi. org/10.1023/A:1016279602893.

Wagner, C., \& Valtin, R. (2003). Noten oder Verbalbeurteilungen? Zeitschrift für Entwicklungspsychologie und Pädagogische Psychologie, 35(1), 27-36. https://doi.org/10.1026//0049-8637.35.1.27.

Walsen, J. C. (2013). Das Wohlbefinden von Grundschulkindern: Soziale und emotionale Schulerfahrungen in der Primarstufe. Dissertation. Oldenburg: Universität Oldenburg.

Wieczerkowski, W., Nickel, H., Janowski, A., Fittkau, B., Rauer, W., \& Petermann, F. (2016). AFS. Angstfragebogen für Schüler. Göttingen: Hogrefe. 